\title{
Neuronal regeneration in injured rat spinal cord after human dental pulp derived neural crest stem cell transplantation
}

\author{
Kabatas $\mathrm{S}^{1}$, Demir $\mathrm{CS}^{2}$, Civelek E ${ }^{1}$ Yilmaz $\mathrm{I}^{2}$, Kircelli A ${ }^{4}$, Yilmaz C $\mathrm{C}^{4}$, Akyuva $\mathrm{Y}^{1}$, Karaoz E $\mathrm{E}^{2,3}$ \\ University of Health Sciences, Gaziosmanpaşa Taksim Training and Research Hospital, Department of Neurosurgery, \\ Karayollari Mahallesi, Gaziosmanpasa, Istanbul, Turkey. kabatasserdar@hotmail.com
}

\section{ABSTRACT}

OBJECTIVE: This study aimed to analyze the effect of human Dental Pulp-Neural Crest Stem Cells (hDP-NCSCs) delivery on lesion site after spinal cord injury ( $\mathrm{SCl}$ ), and to observe the functional recovery after transplantation. METHODS: Neural Crest Stem Cells (NCSCs) were isolated from human Dental Pulp (hDP). The experimental rat population was divided into four groups $(n=6 / 24)$. Their behavioral motility was scored regularly. After 4-weeks, rats were sacrificed, and their spinal cords were examined for Green Fluorescent Protein (GFP) labeled hDP-NCSCs by immunofluorescence (IF) staining.

RESULTS: In early post-injury (p.i) period, the ultrastructure of spinal cord tissue was preserved in Group 4. The majority of cells forming the ependymal region around the central canal were found to be hDP-NCSCs. While the grey-and-white-matter around the ependymal region was composed of e.g. GFP cells, with astrocytic-like appearance. The scores showed significant motor recovery in hind limb functions in Group 4. However, no obvious change was observed in other groups.

CONCLUSION: Cells e.g., mesenchymal (Vimentin+) which express GFP+ cells in the gray-and-white-matter around the ependymal region could indicate the potential to self-renewal and plasticity. Thus, transplantation of hDP-NCSCs might be an effective strategy to improve functional recovery following spinal cord trauma (Fig. 10, Ref. 32). Text in PDF www.elis.sk.

KEY WORDS: functional recovery, human dental pulp, neural crest stem cells, spinal cord injury.

\begin{abstract}
Abbreviations: BBB-scoring - Basso, Beattie and Bresnahan scoring, BDNF - Brain derived neurotrophic factor, BFGF - Basic fibroblast growth factor, CNS - Central nervous system, CNPase - 2',3'-cyclic-nucleotide 3'-phosphodiesterase, CNTF - Ciliary neurotrophic factor, DAPI - 4',6-diamidino-2-phenylindole, DMEM - Dulbecco's modified Eagle's medium, DPSCs - Dental pulp stem cells, EGF - Epidermal growth factor, EP3 - Prostoglandin E2 receptor, FBS - Fetal bovine serum, GDNF - Glial cell-derived neurotrophic factor, GFAP - Glial fibrillary acidic protein, GFP - Green fluorescent protein, hDP - human Dental pulp, hDPSCs - human Dental pulp stem cells, hDP-NCSCs - human Dental pulp-neural crest stem cells, HE - Hematoxylin\&Eosin, IF - Immunofluorescence, L+T - Laminectomy and trauma, MHC-1 - Major histocompatibility complex 1, ms - Milliseconds,
\end{abstract}

${ }^{1}$ University of Health Sciences, Gaziosmanpasa Taksim Training and Research Hospital, Department of Neurosurgery, Istanbul, Turkey, ${ }^{2}$ Liv Hospital, Center for Regenerative Medicine and Stem Cell Research\&Manufacturing (LivMedCell), Istanbul, Turkey, ${ }^{3}$ University of Istinye, Faculty of Medicine, Department of Histology\&Embryology, Istanbul, Turkey, and ${ }^{4}$ Baskent University School of Medicine, Department of Neurosurgery, Ankara,Turkey

Address for correspondence: S. Kabatas, MD, University of Health Sciences, Gaziosmanpasa Taksim Training and Research Hospital, Department of Neurosurgery, Karayollari Mahallesi, Osmanbey Caddesi 616, Sokak No: 10, 34255 Gaziosmanpasa, Istanbul, Turkey.

Phone: +90.212.9453000-3628, Fax: +90.212 .9453180$
MSC - Mesenchymal stem cell, NCSCs - Neural crest stem cells, $\mathrm{NF}$ - Neural factor, NGF - Nerve growth factor, PBS - Phosphatebuffered saline, PFA - Paraformaldehyde, pGFP - plasmid Green fluorescent protein, p.i. - Postinjury, RT - Room temperature, SCI - Spinal cord injury, V - Volts, VEGF - Vascular endothelial growth factor

\section{Introduction}

Spinal cord injury (SCI) has many distinct factorial aspects including primary mechanical damage, secondary cell apoptosis, reactive gliosis, and axons inability to regenerate (1-3). Due to the non-responsive environment of the injured spinal cord, axon regeneration does not occur (4). Besides, the loss of function after SCI might be from both the primary mechanical insult and the subsequent, multifaceted secondary degenerative response. However, some experimental studies in last decades proved that the injured spinal cord could be restored (5-9). Nowadays, stem cell based therapy is promising some valuable strategies for functional recovery of the injured spinal cord (10). In this content, human dental pulp-neural crest stem cells (hDP-NCSCs) were used in addition to neural progenitor stem cells for functional recovery in SCI (11). hDPSCs are neural crest-derived stem cells residing within the perivascular niche of the dental pulp. hDPSCs appear to be an excellent source of stem cells because they can be obtained without adverse health effects and can be obtained 


\section{3-151}

non- invasively from extracted teeth discarded as medical waste, avoiding many ethical issues. hDPSCs show self-renewal ability, immunomodulatory capacity, and proliferation in vitro, and they can differentiate into muscle, cartilage, bone, and other cell types in vitro and in vivo $(12,13,14)$. In this study, it was aimed to analyze the healing effect of hDP -NCSCs on lesion site after SCI and to observe the degree of functional recovery in motility after transplantation.

\section{Materials and methods}

\section{Animals}

The SCI study included about 2 months old 24 female, nonpregnant Wistar albino rats with a weight of 200-300 g. Rats were divided into four groups $(n=6 / 24)$ : only laminectomy (Group 1); laminectomy + trauma (L+T) (Group 2); $\mathrm{L}+\mathrm{T}+$ phosphate-buffered saline (PBS) (Group 3); L+T+hDP-NCSCs (Group 4), respectively. Rats were sacrificed 4 weeks after transplantation. The experimental design and all procedures were approved by the Ethics Committee of Kocaeli University.

\section{Isolation and culture of hDP-NCSCS}

To isolate hDP-NCSCs, normal human third molars $(\mathrm{n}=5)$ were obtained from adults (17- to 25-yr-old) at the Dental Hospital of Kocaeli University according to the guidelines provided by ethics committee. Isolation and culture of hDP-NCSCs were performed according to protocols described elsewhere $(15,16)$. Briefly, the pulp tissue of each sample was gently separated from the crown and root, digested with collagenase type I (Sigma Aldrich, St. Louis MO) to generate single cell suspensions. To culture the isolated NCSCs derived from dental pulp; MEM-Earle (Biochrom-FG0325) medium was used including $10 \%$ fetal bovine serum (FBS; Invitrogen/ GIBCO, Grand Island, NY, USA) and $100 \mathrm{IU} / \mathrm{mL}$ penicillin-100 $\mu \mathrm{g} / \mathrm{mL}$ streptomycin (P/S; Invitrogen/ GIBCO). Cells were cultured in tissue culture flasks and incubated at $37{ }^{\circ} \mathrm{C}$ in a humidified atmosphere containing $5 \% \mathrm{CO}_{2}$. The adherent cells were grown to passage three (P3). At passage three, cells were used for characterization and differentiation studies.

\section{Characterization of hDP-NCSCs by flow cytometry}

Undifferentiated hDP-NCSCs at P3 were subjected to flow cytometry analysis [FACSCalibur (BD Biosciences, San Jose, CA)]. Immunophenotyping analysis was performed for these antigens: CD45/34/11b/ MHC Class-II/19, CD44, CD73, CD90 and CD105 (BD Bioscience).

\section{In vitro differentiation of $h D P-N C S C S$}

To induce adipogenic differentiation, cells were seeded onto 6-well plates (P3; 3000 cells $\left./ \mathrm{cm}^{2}\right)$ and cultured with Mesenchymal stem cell (MSC) Basal Medium (StemCell Technologies Inc., Vancouver, BC, Canada) supplemented with $10 \%$ adipogenic supplement and $1 \% \mathrm{P} / \mathrm{S}$ for 3 weeks. The medium was refreshed every 3-4 days. The differentiated cells were stained with $0.5 \%$ oil red O (Sigma-Aldrich) to confirm the formation of intracellular lipid droplets, which indicates adipogenic differentiation.
For osteogenic differentiation, cells (P3; 3000 cells $\left./ \mathrm{cm}^{2}\right)$ were seeded on collagen precoated cover slips in 6-well plates. The differentiation medium (LDMEM supplemented with $0.1 \mu \mathrm{M}$ dexamethasone (Sigma-Aldrich), $0.05 \mu \mathrm{M}$ ascorbate2-phosphate (Wako Chemicals, Richmond, VA, USA), $10 \mathrm{mM}$ $\beta$-glycerophosphate (Sigma-Aldrich), $2 \%$ primocin (Invivogen) and $10 \%$ FBS) was replaced twice a week. After 4 weeks, osteogenic differentiation was estimated by alkaline phosphatase activity. Cells were fixed for $5 \mathrm{~min}$ in ice-cold $70 \%$ ethanol. The cells were stained with alizarin red S (2\%, pH 4.2). Stained cells were dehydrated in pure acetone, fixed in acetone-xylene (1:1) solution, cleared with xylene.

To induce neurogenic differentiation, cells were cultured for 3-5 days in differentiation medium (DMEM supplemented with $0.5 \mathrm{mM}$ isobutylmethylxanthine, $10 \mathrm{ng} / \mathrm{ml}$ brain-derived neurotrophic factor (BDNF), $10 \mathrm{ng} / \mathrm{ml}$ epidermal growth factor (EGF), $10 \mathrm{ng} / \mathrm{ml}$ basic fibroblast growth factor (BFGF), $20 \%$ neural stem cell proliferation supplement (StemCell Technologies Inc.) and 1 $\%$ penicillin-streptomycin. The cells stained antibodies which are associated with neural like cells (GFAP/c-fos, NF/c-fos, Nestin/cfos and CNTF/BDNF).

\section{Green fluorescent protein (GFP) labeling of hDP-NCSCs}

The pGFP plasmid was obtained from Clontech (Palo Alto, CA), amplified in XL-1 strain of E. coli and purified using an endotoxin-free plasmid isolation kit (Qiagen). The plasmid was transfected into the target cells using a Neon Transfection System (Invitrogen, Carlsbad, CA). In total, $2 \times 10^{5}$ target cells were mixed with $1 \mu \mathrm{g}$ plasmid DNA in $10 \mu \mathrm{l}$ transfer buffer that was supplied with the transfection system kit (Invitrogen). The transfection parameters were adjusted to 1200 volts (V), 40 milliseconds (ms), and a single pulse. The transiently transformed cells were transferred into a tube containing $1 \mathrm{ml}$ DMEM supplemented with 10 $\%$ FBS. Following $48 \mathrm{~h}$ of incubation, the cells were selected with G418 (Gibco, $200 \mu \mathrm{g} / \mathrm{ml}$ in media) for 6 weeks.

\section{Surgical procedure and cell transplantation}

For skin preparation of T10-11 spinal cord surgery, dorsal laminectomy of tracer injection, dermal surface of the related regions was cleared by hair razor, and the skin was washed by antibacterial soap followed with betadine and $70 \%$ ethanol application (17). After an overnight fast with unrestricted access to water, all 24 rats were anesthetized with intramuscular ketamine $(50 \mathrm{mg} / \mathrm{kg})$ and xylazine $(5 \mathrm{mg} / \mathrm{kg})$ prior to surgery. Under dissection stereo microscope; $3 \mathrm{~mm}$ long laminectomy, encompassing the caudal end of T10 vertebra and the rostral end of T11 vertebra, was performed. For SCI groups (total: $\mathrm{n}=18$ ), a severe T10-T11 contusive injury was introduced by dropping the impounder rod $(1 \mathrm{~g})$ from a height of $50 \mathrm{~mm}$. GFP labeled hDP-NCSCs $\left(3 \times 10^{5}\right.$ cells $/ 5 \mu \mathrm{L}$ ) were transplanted into the injured spinal cord via Hamilton syringe (Hamilton company, Reno, NV) connected to a syringe pump (KD Scientific Inc., Holliston, MA, USA) for $5 \mathrm{~min}$, respectively. PBS group received $5 \mu \mathrm{L}$ of PBS at the injured spinal cord with the same technique. The needle was removed $10 \mathrm{~min}$ after subcutaneous transplantation, and muscle \& skin layers were 
closed in layers. The urinary bladders of SCI rats were evacuate twice daily during the entire study.

\section{Functional tests}

Functional tests were performed using the Basso, Beattie and Bresnahan (BBB) locomotor rating scale at pre-surgery, at days 1, $7,14,21$, and 28 postinjury (p.i.). Two independent, blinded examiners observed each animal for $4 \mathrm{~min}$. Hind limb movements were recorded by video camera and locomotor functions were assessed (18). The BBB scores were presented as mean \pm standard error.
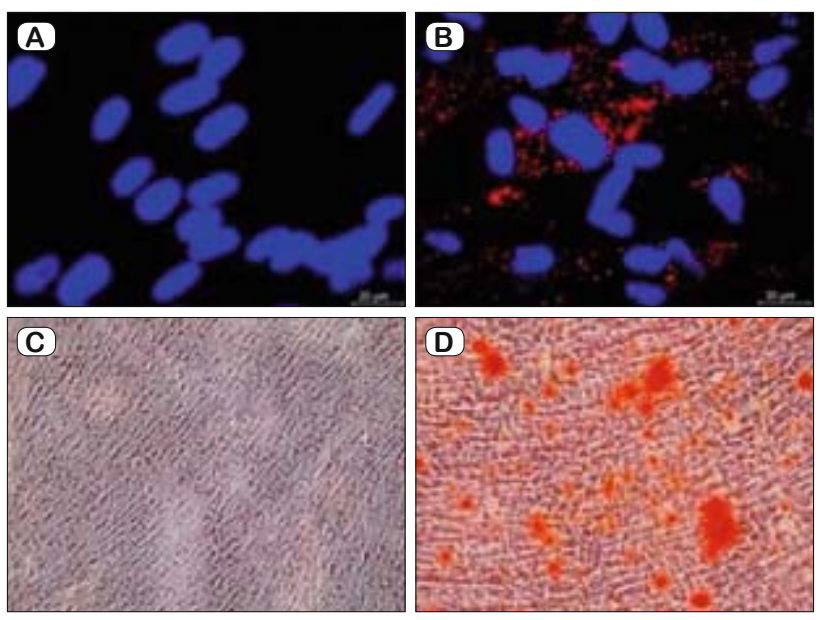

(E)
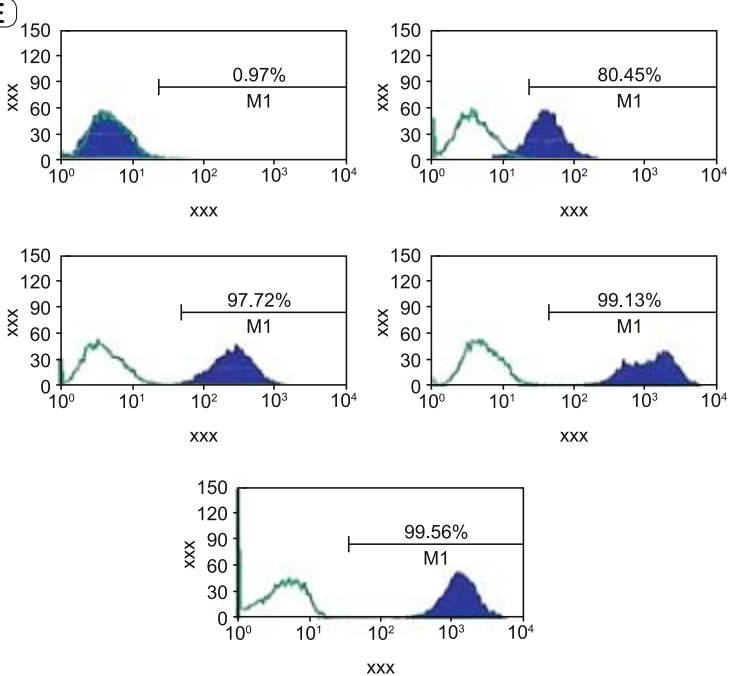

Fig. 1. Characterization of hDP-NCSCs. The cells differentiated into the adipogenic lineage after 15 days of incubation and osteogenic differentiation (day 8) after osteogenic induction. Adipogenic differentiation was identified by neutral lipid vacuole formation (stained with Oil Red O, A - Control, B - Differentiated hDP-NCSCs). Mineral nodules were stained positive with Alizarin Red S stain (C - Control, D - Differentiated hDP-NCSCs). Immunophenotypic properties of hDP-NCSCs evaluated with flow cytometry (E). Predefined markers that specified MSCs were used to define the characteristics of cultured cells. The hDP-NCSCs expressed all MSC markers including; CD44, CD90 and CD105 but not CD45, CD34 or MHC class II (Scale bars: A,B-20 $\mu \mathrm{m} ; \mathrm{C}, \mathrm{D}-100 \mu \mathrm{m})$.
Tissue harvesting and immunohistochemical examination

To detect GFP + hDP-NCSCs, an immunofluorescence double staining protocol was performed on the paraffin-embedded tissues (19). Slides were deparaffinized with two changes of xylene for 5 min each and then rehydrated in a series of graded alcohol solutions of 100,90 , and $80 \%$. Antigen retrieval was performed on the slides using a steamer-citrate buffer antigen retrieval method. Endogenous peroxidase activity was blocked by incubating the slides in fresh $3 \% \mathrm{H}_{2} \mathrm{O}_{2}$ in PBS buffer. Nonspecific staining was blocked with a mixture of two different sera at $1.5 \%$ in PBS for 30 min at room temperature (RT). The sections were incubated for 1 $\mathrm{h}$ at RT with the following primary antibodies: anti-GFP antibody (sc-9996; Santa Cruz, Heidelberg, Germany), Vimentin (sc-7557; Santa Cruz), GFAP (MS-280-P, Thermo Scientific, Rockford, IL), BDNF (sc-20981; Santa Cruz), Nestin (SC-33677; Santa Cruz) and NF (SC-12980; Santa Cruz). Also the sections stained for proinflammatory cytokines such as; MPO (SC-34161; Santa Cruz), IL-1 $\beta$ (SC-23460; Santa Cruz), IL-6 (SC-1265; Santa Cruz), MIP2 (SC-1388; Santa Cruz) and anti-inflammatory cytokines such as IL-1ra (SC-25444; Santa Cruz) and EP3 (SC-16019; Santa Cruz). The sections were incubated in a mixture of two fluorescenceconjugated secondary antibodies, which included goat anti-mouse FITC (SC-2010; Santa Cruz), donkey anti-goat TR (SC-2783; Santa Cruz), and goat anti-mouse TR (SC-2781; Santa Cruz) at a dilution of 1:50 in PBS for $30 \mathrm{~min}$ at RT. The sections were mounted with mounting medium containing DAPI (Santa Cruz Biotechnology). Then, the mounted cells were examined under a fluorescence microscope (Leica DMI 4000B, Wetzlar, Germany).

\section{Statistical analyses}

Statistical analysis was performed with SPSS software for Windows (SPSS for Windows, Version 21, SPSS Inc, Armonk, NY, USA). Descriptive analyses were presented using means and standard deviation for normally distributed variables. Univariate parametric variance analysis was used for groups which did not fulfill the requirements of parametric test (ANOVA). Nonparametric data were analyzed with Kruskall - Wallis test. In order to investigate differences between the groups, Tukey test was used for parametric and Dunn test was used for nonparametric data. Statistical significance was defined by $\mathrm{p}<0.05$.

\section{Results}

Culture and characterization of hDP-NCSCs

hDP-NCSCs attached to the culture flasks sparsely and nonadherent small cells presented in the primary culture were gradually depleted in further passages. Following 3-4 days of incubation, proliferation started and the cells gradually grew into small colonies. A pure population of fibroblast-like, spindle-shaped cells appeared after three passages. The expression of hDP-NCSCs markers was analyzed by flow cytometry, revealing cells that were positive for CD44, CD73, CD90 and CD105 and negative for CD45/34/11b/ major histocompatibility complex (MHC) Class-II/19 (Fig. 1 A-E). The multipotency of the isolated cells was determined by adipogenic, neurogenic and osteogenic dif- 

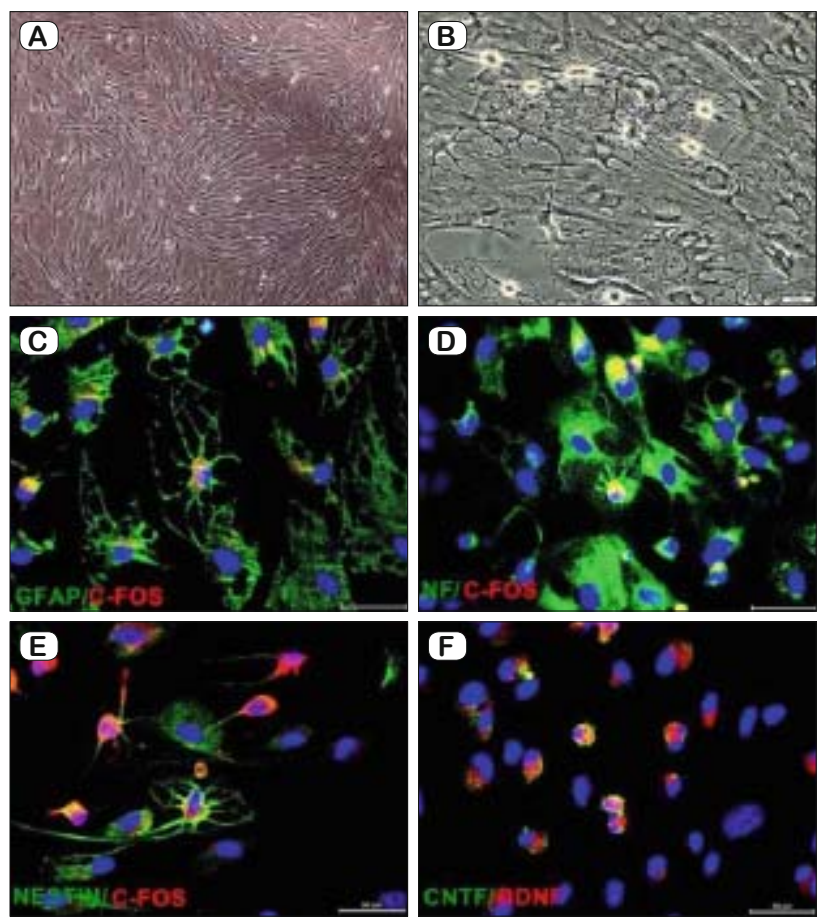

Fig. 2. Differentiation of hDP-NCSCs into neuron-glial like cells in-vitro and expression of neural markers. Phase contrast image after 5 days; neuron-like cells displayed distinct morphologies (A - Control, B - Morphology of neural differentiated cells). Differentiated cells represented intensified staining of C - GFAP (green) and c-fos (red); D - Neurofilament (green) and c-fos (red); E - Nestin (green) and c-fos (red); F - CNTF (green) and BDNF (red) (Scale bars: $50 \mu \mathrm{m}$ ).

ferentiation. After three weeks of culture, hDP-NCSCs in the adipogenic differentiation cocktail developed enlarged lipid droplets that invaded the entire cytoplasm of each cell. Their differentiation into adipocytes was confirmed by Oil Red O staining (Fig. 1 B).
Moreover, the cells cultured in the osteogenic differentiation medium formed cellular aggregates that were characterized by the presence of amorphous material deposits. The amorphous deposits were observed under a microscope. Calcium deposits were stained by Alizarin Red S (Fig. 1 D). The neurogenic differentiation of isolated cells was observed in 5-day-old culture media that included a chemical cocktail. Neuron-like cells displayed distinct morphologies that ranged from extensively simple, bipolar cells to large, branched and multipolar cells. These cells positively stained for some neuron or glial cell-specific markers, including GFAP/c-fos, NF/c-fos, Nestin/c-fos and CNTF/BDNF (Fig. 2 A-F).

\section{Survival and migration of $h D P-N C S C s$}

After 4 weeks, the immunofluorescence microscopic analyses of longitudinal and transversal sections of rat spinal cords from experimental groups were performed with double staining of GFP together with Nestin, GFAP, BDNF, Vimentin, NF, S100. In all sections of 1st, 2nd and 3rd groups, they showed negative staining for GFP. Noticeably, the center of the lesion was severely cavitated in the 2nd and 3rd groups (Figs 3, 4, 5, 6 and 7). However, GFP+ cells were observed in the vicinity of the damage site of Group 4 at the end of 4 weeks, and most of the surviving cells were located at the periphery of the lesion site next to healthier tissue (Figs 3, 4, 5, 6 and 7). GFP + hDP-NCSCs migrated into cavitated area from the injection sites, and the majority of these still survived and expressed some stem cell and neural markers such as such as GFAP (Fig. 3), nestin (Fig. 4), NF (Fig. 5), BDNF (Fig. 6) and vimentin (Fig. 7). Interestingly, the sections of low scored animals of Group 4 in tests showed considerably lower number of GFP+ cells migrated to the damage site than the sections of higher scored animals. Additionally, the increase of GFAP expression in this region is remarkable (Fig. 3).

Tissue sections were also stained for pro-inflammatory (MPO, IL-1 $\beta$, IL-6 and MIP-2) and anti-inflammatory cytokines (IL-1ra
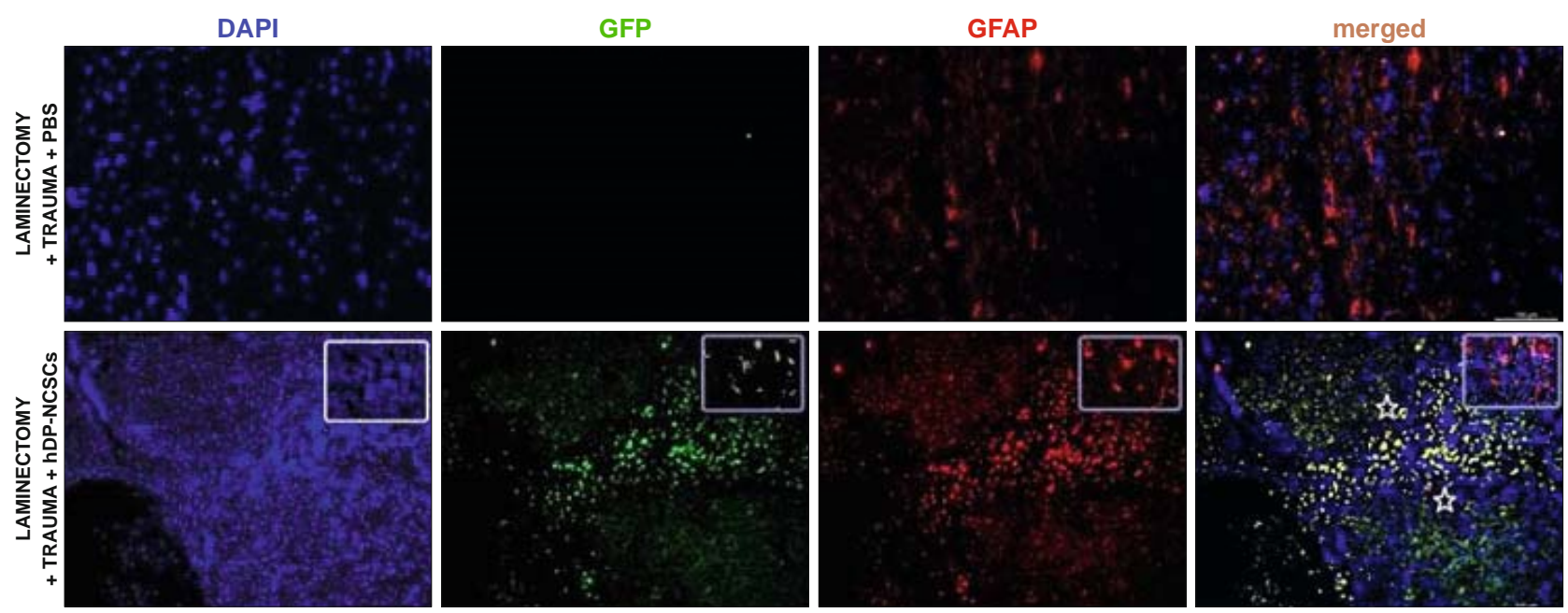

Fig. 3. The immunostaining of longitudinal sections of rat spinal cords for GFAP expression. GFAP is expressed by numerous cell types of the CNS; its different expression levels in the tissue sections may indicate neuronal regeneration. In the $3^{\text {rd }}$ group; GFAP expression was weak. By contrast, the $4^{\text {th }}$ group demonstrated strong staining patterns in the lesion area (stars), indicating that the hDP-NCSCs might be directly involved in differentiation-mediated regeneration. The nucleus of cells was stained with DAPI (blue) (Scale bars: $100 \mu \mathrm{m}-200 \mu \mathrm{m})$. 

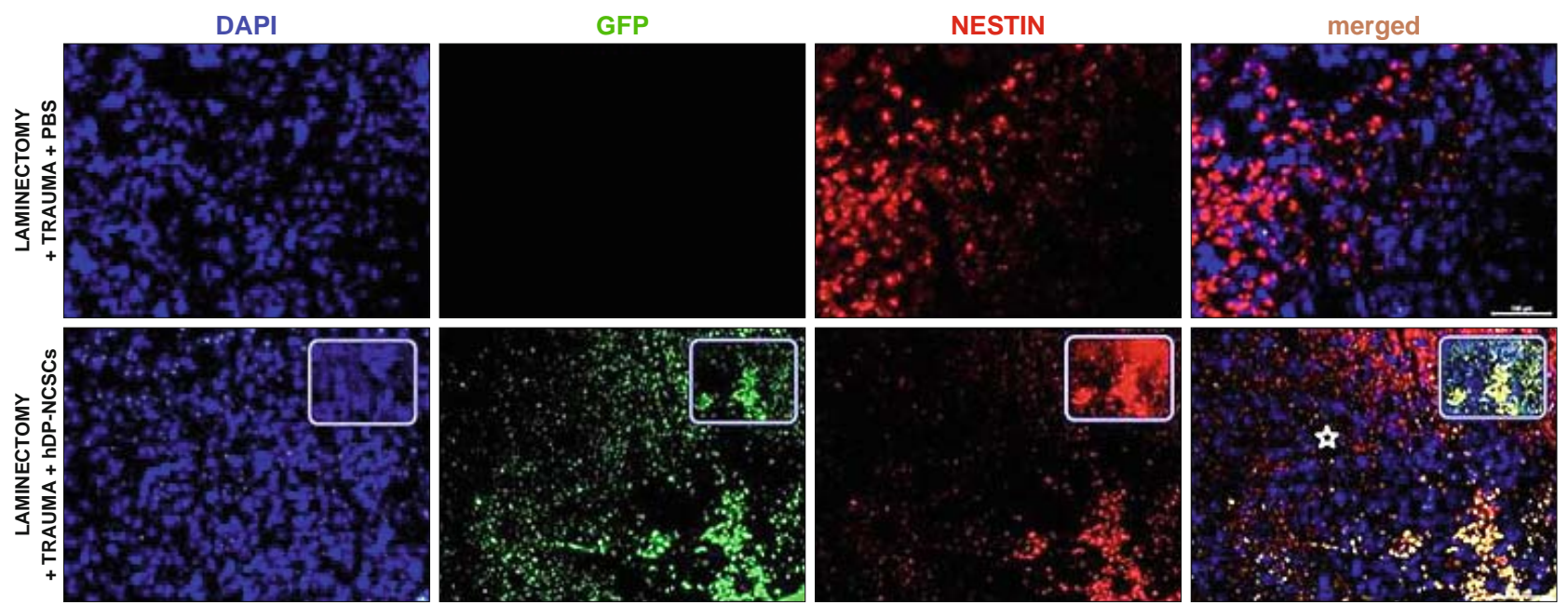

Fig. 4. Detection of Nestin positive cells in the SCI lesion site (star). The expression of Nestin - a protein marker for neural stem cells - was induced in the tissue by the signals derived p.i. The expression level of Nestin was limited in the $3^{\text {rd }}$ group. By contrast, the expression of Nestin by GFPpositive hDP-NCSCs near the lesion epicenter increased significantly. The nucleus of cells was stained with DAPI (blue) (Scale bars: $100 \mu \mathrm{m}$ ).
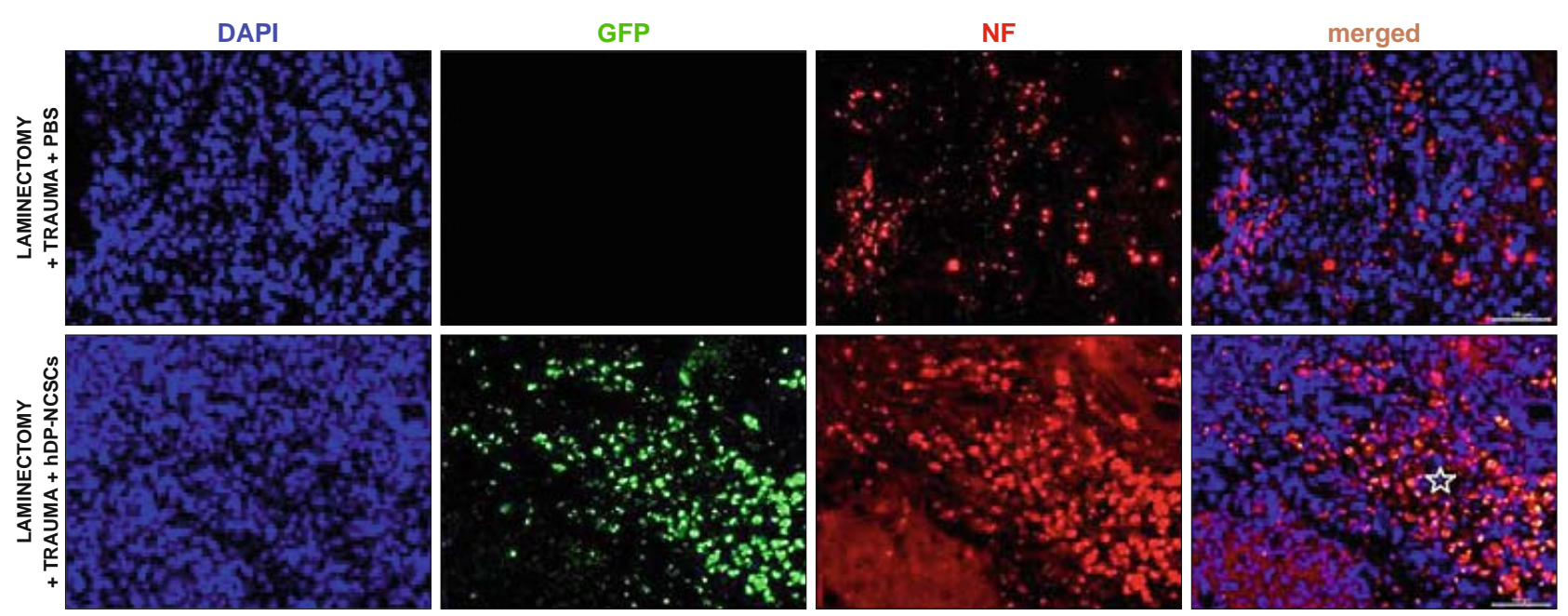

Fig. 5. The IF staining of Neurofilament in the SCI lesion site (star). Neurofilament is a major component of the neuronal cytoskeleton, and believed to function primarily to provide structural support for the axon and to regulate axon diameter. In the $4^{\text {th }}$ group, expression of Neurofilament was significantly increased than in the $3^{\text {rd }}$ group. The nucleus of cells was stained with DAPI (blue) (Scale bars: $\left.100 \mu \mathrm{m}\right)$.

and EP3) (Fig. 8). After 4 weeks of stem cell injection, staining of the tissue sections with pro-inflammatory and anti-inflammatory cytokines showed different statistical results. In Group 4, the expression of pro-inflammatory cytokines decreased compared to the other groups $(\mathrm{p}<0.05)$, and the expression of anti-inflammatory cytokines significantly increased $(\mathrm{p}<0.05)$ (Fig. 9).

\section{Functional recovery}

To confirm the traumatic impact of the standardized severe weight-drop contusion injury to the T10-T11 spinal cord, the hind limb locomotion of the SCI rats was evaluated. At each assessment time point, consistent functional deficits were noted among SCI rats with the BBB locomotion scores showing profound loss initially, which was then gradually improved and approaching a plateau level of spontaneous recovery typical for this type of injury by 4 weeks p.i. According to BBB locomotor activity test the performances of the Group 4 were statistically different than of the Group 2 and $3(p<0.05)$. The injured rats (Group 2 and 3) showed marked lower activity score than MSC injected group (Group 4) in the BBB locomotor rating score. All experimental groups (Group 2-4) showed BBB locomotion score increment to some extent, but the highest scores were observed in the MSC injected group (Group 4) (Fig.10).

\section{Discussion}

MSC shows its therapeutic effect not only by transdifferentiating into necessary tissue cells in the damaged region, but also 

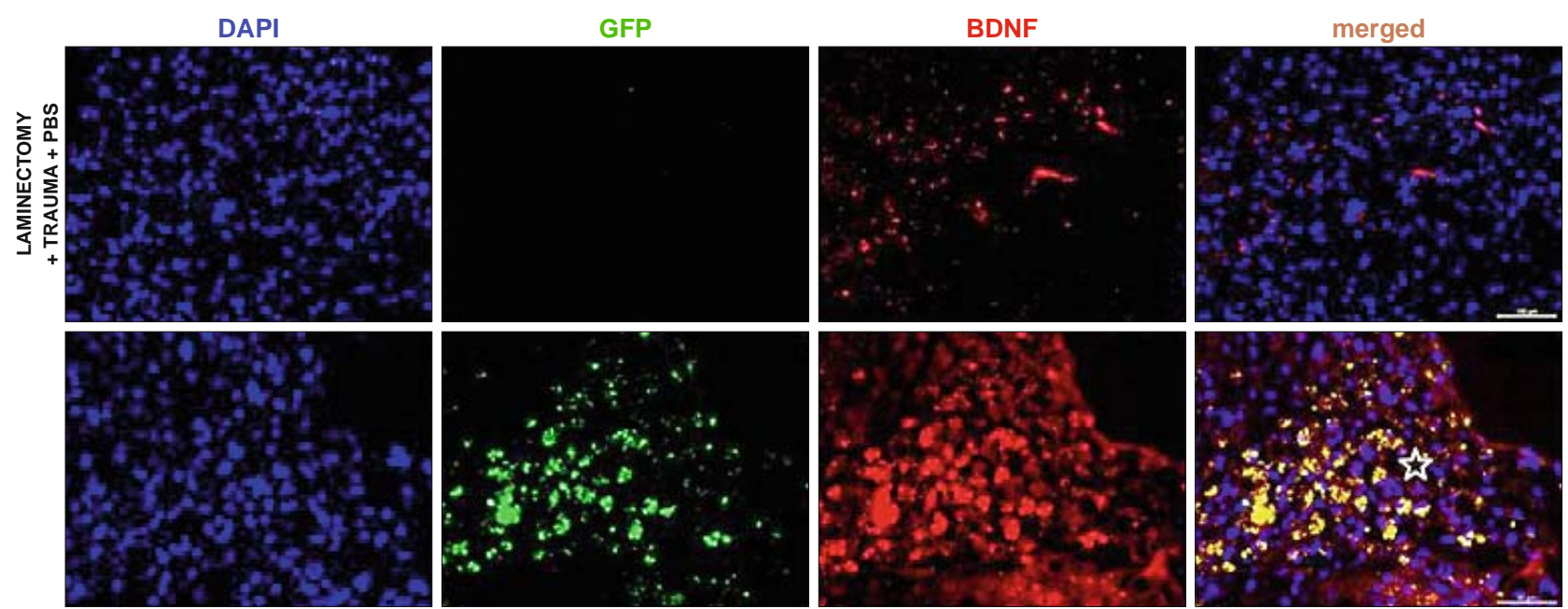

Fig. 6. Detection of BDNF in the SCI lesion site (star). The expression of this NF was induced in the tissue by the signals derived post injury. The expression level of BDNF was limited in the $3^{\text {rd }}$ group. In contrast, the expression of BDNF by GFP-positive hDP-NCSCs near the lesion epicenter increased significantly, which might support axonal outgrowth. The nucleus of cells was stained with DAPI (blue) (Scale bars: $100 \mu \mathrm{m}, 50 \mu \mathrm{m})$.
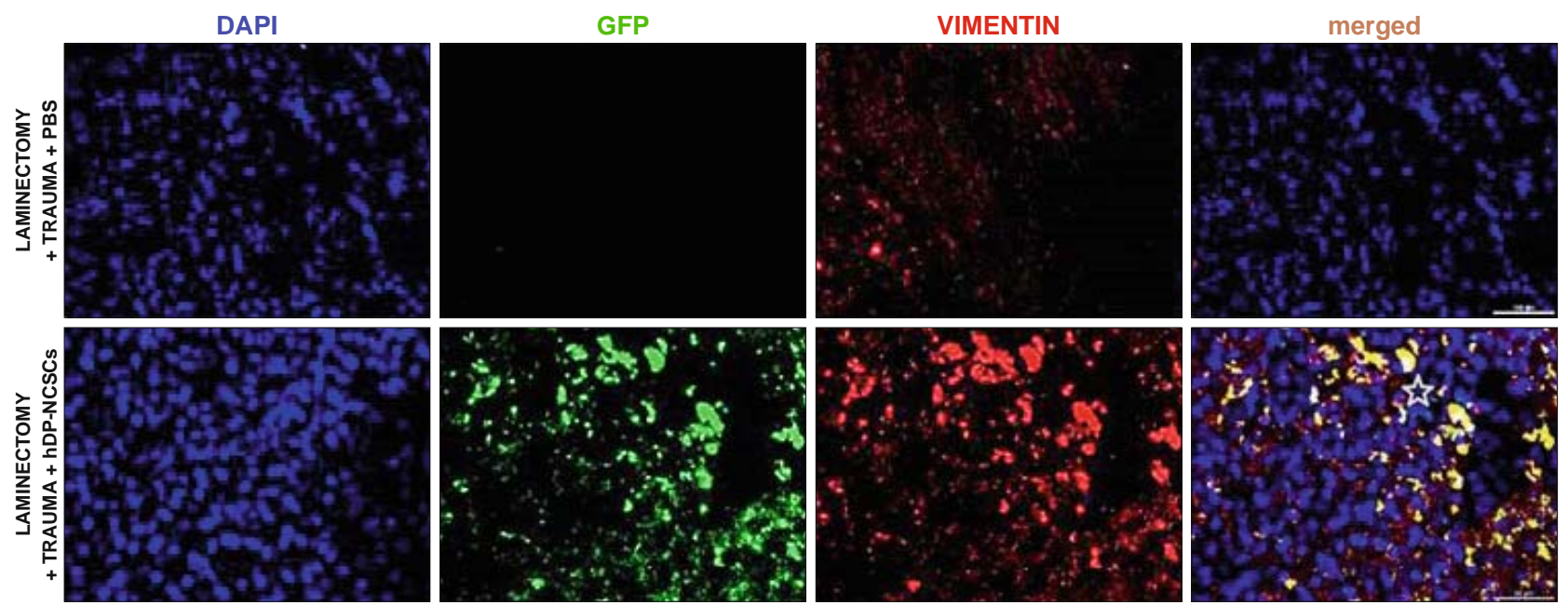

Fig. 7. The infiltration of hDP-NCSCs into the lesion site. The stem cells transplanted into the spinal cord were labeled previously with GFP. Vimentin was used as an MSC marker and was used along with GFP to track the hDP-NCSCs in the tissue. The GFP/vimentin-positive cells collected around the lesion site (star) in the hDP-NCSCs -transplanted tissues at 28 days p.i. The nucleus of cells was stained with DAPI (blue) (Scale bars: $100 \mu \mathrm{m}, 50 \mu \mathrm{m}$ ).

by increasing the growth factor and cytokine secretions, which have different paracrine and autocrine activities in the damaged region. However, it also has immunomodulatory and anti-inflammatory effects $(13,20)$. Cytokines are an important regulator of inflammation following acute SCI (21). Regarding the IF staining results in our study, anti-inflammatory cytokines (IL-1 ra and EP3) expressions were higher in the 4th group than other groups. Increase in anti-inflammatory cytokines indicated that the damaged area was repaired. The reduction in the inflammatory cytokines (MPO, IL-1 $\beta$, IL-6 and MIP-2) also supports this result. IL-1 $\beta$ has been implicated as a pro-inflammatory cytokine, whose inhibition may prove to be beneficial in SCI (22). The levels of IL-1 $\beta$ in the 4th group significantly decreased. IL-6 remains a cytokine with dual functions. On the one hand, it has been demonstrated to modulate the immune response by increasing inflammation after SCI. High levels of IL-6 can shift NSC differentiation toward astrocytes rather than neurons, thus promoting glial scar formation (23). Additionally, suppression of IL-6 levels reduces secondary injury by reducing inflammatory cell infiltration and may result in improved recovery after SCI $(24,25)$. Moreover, a medium level of IL-6 is important for preserving neurogenesis and neuroprotection following central nervous system (CNS) injuries (26). In the present study, hDP-NCSCs partially decreased IL-6 levels, while high levels of IL-6 were observed in the other groups. 


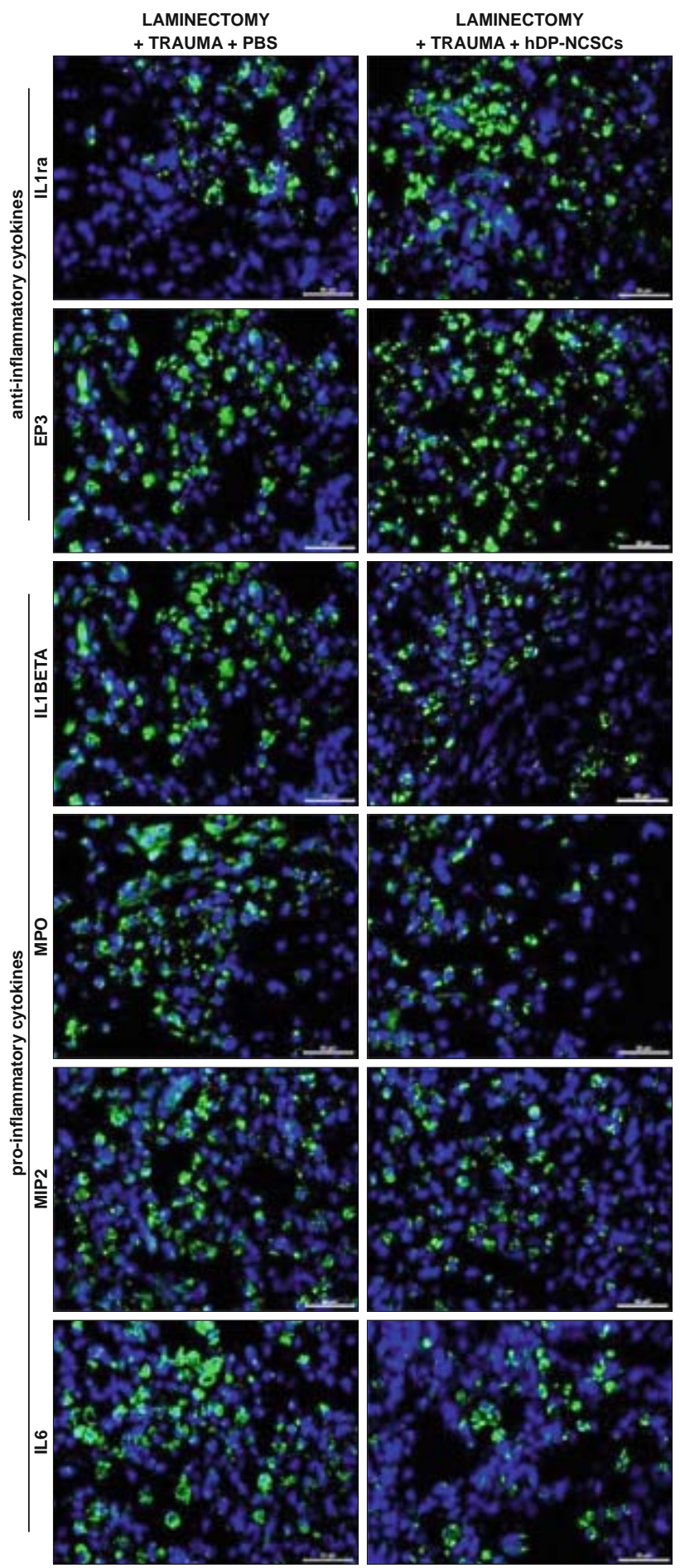

Fig. 8. The IF staining of paraffin-embedded spinal cord tissue sections for IL1 ra, EP3, IL1ß, MPO, MIP2 and IL6 after transplantation in Group 3 and 4. The density of IL1ß, MPO, MIP2 and IL6 positive cells significantly decreased and IL1 ra, EP3 expression was increased after hDP-NCSCs injection. The nucleus of cells was stained with DAPI (blue) (Scale bars: $50 \mu \mathrm{m}$ ).
Previous studies reported that hDPSCs display an increased level of immunosuppressive activities when compared with Bone Marrow-Mesenchymal Stem Cells (BM-MSCs). Huang et al. showed that the implantation of DPSCs derived from rhesus monkeys into the hippocampus of mice did not cause any immune rejection. Similarly, higher expressions of human MHC-1, which indicates a stronger degree of immunosuppression, were seen in mouse livers, which were incorporated with hDPSCs (14). In the present study, an acute host immune rejection, such as major infiltration of lymphocytes, directed toward the transplanted cells was not observed (27). The IF results at 4th week showed that parts of implanted MSCs have transformed into nerve cells and can survive for long time. They basically adapt to the body's environment and continue to promote the recovery of the injured spinal cord function. The Hematoxylin\&Eosin (HE) staining results of 1st week and 4th week showed that immune rejection of MSCs transplantation was not significantly different from the group of non-implanted cells.

In vitro studies have shown that hDPSCs can differentiate toward functionally active neurons under appropriate culture conditions (14). In this study, we determined that hDP-NCSCs could be isolated and amplified effectively in vitro. It could grow well in the special medium for MSCs. Redundant MSCs can be obtained through passage, which can provide experimental foundation for future studies and clinical applications.

Meanwhile, our results showed that hDP-NCSCs transplantation for the treatment of rat SCI can significantly improve the neurological function of the damaged spinal cord at 2 weeks after treatment. And at 4 weeks after the treatment, the improvement was much more significant, which was consistent with the results of previous researches on treatment of SCI by transplantation of MSCs (28). This indicated that the damaged spinal cord could be recovered and regenerated by the implantation of hDP-NCSCs. The mechanism still remains unclear on how the transplanted MSCs survive, concentrate and migrate to the damaged spinal cord. Our findings suggested that at 2 weeks after hDP-NCSCs transplantation into the damage spinal cord, MSCs begin to adapt to the microenvironment of the spinal cord and spontaneously secrete or induce other cells to produce nerve repair factors to promote regional nerve repair and stimulate the surviving nerve axons to stretch out lateral branches to the damaged axons. Then, spinal cord function was improved $(11,29,30)$. It also may be associated with the integration of transplanted cells to the neural circuits $(31,32)$.

\section{Conclusion}

Our study demonstrated that the MSCs can be isolated from the dental pulp and cultured and passaged in vitro. After transplantation of the passaged MSCs into rats with SCI, we found that the isolated MSCs can survive in rat bodies without any immune rejection. The implanted MSCs can differentiate into nerve cells and are involved in the recovery of the damaged spinal cord. Thus, it improves the scores of motion behavior and promotes the recovery of motor function after SCI. These results provide a theoretical and experimental basis for hDP-NCSCs transplantation 
$143-151$
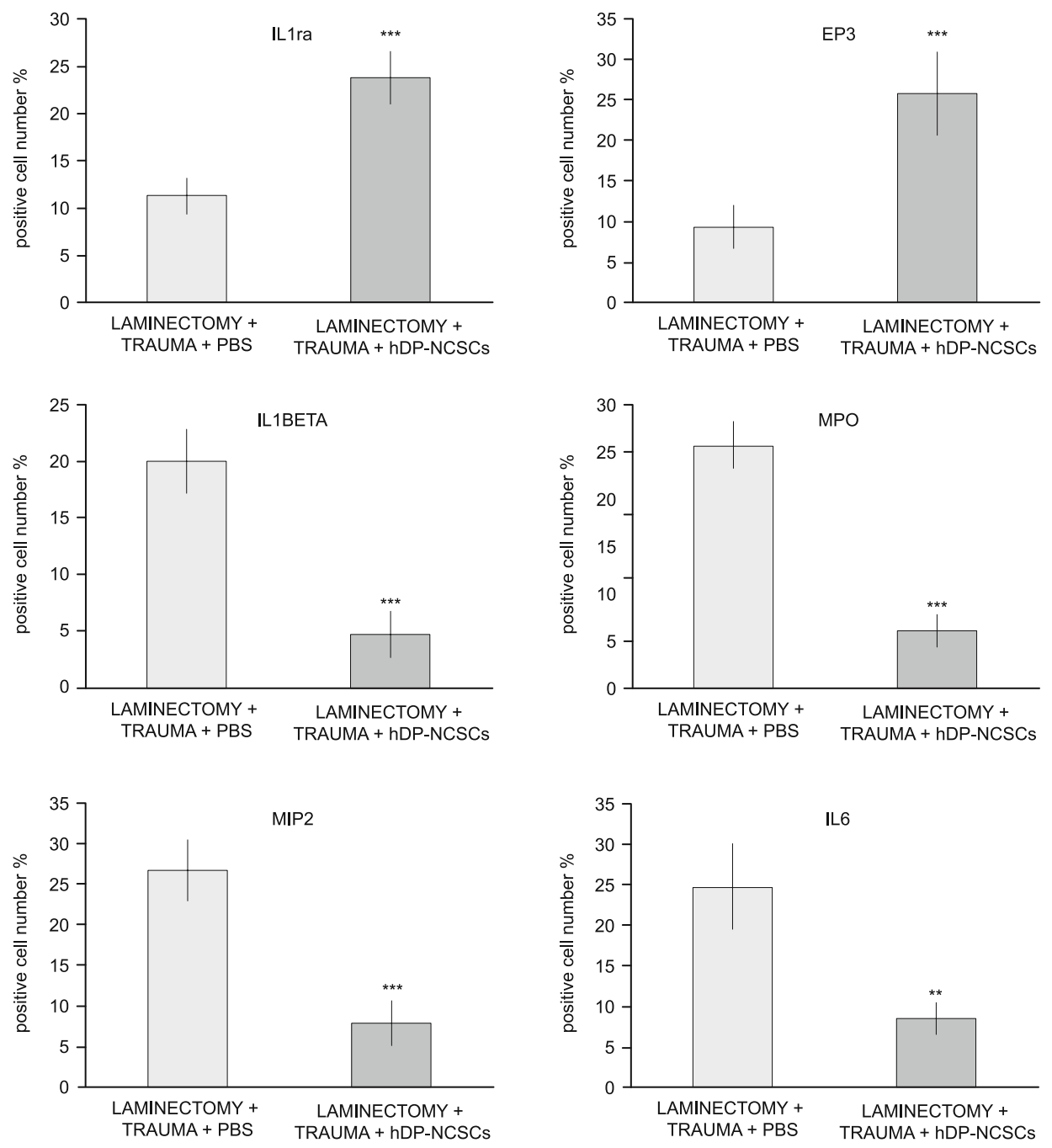

Fig. 9. Quantification of cells expressing proteins associated with inflammation. Stem cell treatment greatly suppressed the expression of the inflammatory markers (IL1ra, EP3, IL-1 $\beta$, IL6, MIP-2 and MPO) in the tissue. After stem cell treatment, cells expressing pro-inflammatory proteins (IL-1 $\beta$, IL6, MIP-2 and MPO) decreased and IL1 ra and EP3 positive cells were increased in the tissue (*p; $0.01<p<0.05, * * p ; 0.001$ $<\mathbf{p}<0.01, * * * \mathrm{p} ; 0.001<\mathrm{p})$.

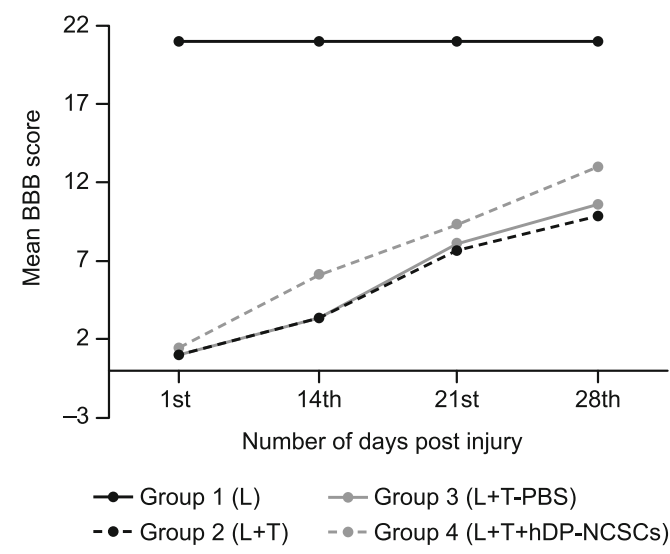

Fig. 10. Effect of T10-11 SCI on general hind limb function over time after SCI. Deficits are expressed as a BBB locomotion score. applied in the treatment of SCI. Compared with other stem cells, hDP-NCSCs have advantages such as good primitiveness, strong amplification ability, simple acquisition, and weak in vivo rejection, without any damage to donor. hDP-NCSCs can provide new cell source and new method for treatment of SCI. As the study is further improved and deepened, it's possible to apply the hDPNCSCs transplantation to clinical treatment of SCI.

\section{References}

1. Teng YD, Kabatas S, Li J, Wakeman DR, Snyder EY, Sidman RL. Functional Multipotency of Neural Stem Cells and Its Therapeutic Implications. In: Ulrich H, editor. Perspectives of Stem Cells: From tools for studying mechanisms of neuronal differentiation towards therapy, 1 st ed. Dordrecht, Heidelberg, London, New York: Springer Science+Business Media B.V.; 2010: 255-270. 
2. Teng YD, Yu D, Ropper AE et al. Functional multipotency of stem cells: a conceptual review of neurotrophic factor-based evidence and its role in translational research. Curr Neuropharmacol 2011; 9 (4): 574-585.

3. Fernández-Martos CM, González-Fernández C, González P, Maqueda A, Arenas E, Rodríguez FJ. Differential expression of Wnts after spinal cord contusion injury in adult rats. PLoS One 2011; 6 (11): e27000.

4. Xie F, Zheng B. White matter inhibitors in CNS axon regeneration failure. Exp Neurol 2008; 209: 302-312.

5. Joosten EA, Veldhuis WB, Hamers FP. Collagen containing neonatal astrocytes stimulates regrowth of injured fibers and promotes modest locomotor recovery after spinal cord injury. J Neurosci Res 2004; 77: 127-142.

6. Karaoz E, Kabatas S, Duruksu G et al. Reduction of lesion in injured rat spinal cord and partial functional recovery of motility after bone marrow derived mesenchymal stem cell transplantation. Turk Neurosurg 2012; 22 (2): 207-217.

7. Sonmez E, Kabatas S, Ozen O et al. Minocycline Treatment Inhibits Lipid Peroxidation, Preserves Spinal Cord Ultrastructure and Improves Functional Outcome after Traumatic Spinal Cord Injury in the Rat. Spine (Phila Pa 1976) 2013; 38 (15): 1253-1259.

8. Aras Y, Sabanci PA, Kabatas $\mathbf{S}$ et al. The Effects of Adipose TissueDerived Mesenchymal Stem Cell Transplantation During the Acute and Subacute Phases Following Spinal Cord Injury. Turk Neurosurg 2016; 26 (1): $127-139$.

9. Cömert S, Altinörs N, Kabataş S et al. Human Mesenchymal Stem Cell Therapy in Thoracic Spinal Cord Injury and Evaluation of the Resultsan experimental study in rats. J Turk Spinal Surg 2017; 28 (2): 83-90.

10. Kabatas S, Teng YD. Potential roles of the neural stem cell in the restoration of the injured spinal cord: review of the literature. Review. Turk Neurosurg 2010; 20 (2): 103-110.

11. Yamamoto A, Matsubara K, Kano F, Sakai K. Analysis of the neuroregenerative activities of mesenchymal stem cells in functional recovery after rat spinal cord injury. Methods Mol Biol 2014; 1213: 321-328.

12. Karaöz E, Demircan PC, Sağlam O, Aksoy A, Kaymaz F, Duruksu G. Human dental pulp stem cells demonstrate better neural and epithelial stem cell properties than bone marrow-derived mesenchymal stem cells. Histochem Cell Biol 2011; 136 (4): 455-473.

13. Özdemir AT, Özgül Özdemir RB, Kirmaz C et al. The paracrine immunomodulatory interactions between the human dental pulp derived mesenchymal stem cells and CD4 T cell subsets. Cell Immunol 2016; 310: $108-115$.

14. Song M, Lee JH, Bae J, Bu Y, Kim EC. Human Dental Pulp Stem Cells Are More Effective Than Human Bone Marrow-Derived Mesenchymal Stem Cells in Cerebral Ischemic Injury. Cell Transplant 2017; 26 (6): 1001-1016.

15. Karaöz E, Doğan BN, Aksoy A et al. Isolation and in vitro characterisation of dental pulp stem cells from natal teeth. Histochem Cell Biol 2010; 133 (1): 95-112.

16. Akpinar G, Kasap M, Aksoy A, Duruksu G, Gacar G, Karaoz E. Phenotypic and proteomic characteristics of human dental pulp derived mesenchymal stem cells from a natal, an exfoliated deciduous, and an impacted third molar tooth. Stem Cells Int 2014; 457059.

17. Choi H, Liao WL, Newton KM et al. Respiratory abnormalities resulting from midcervical spinal cord injury and their reversal by serotonin $1 \mathrm{~A}$ agonists in conscious rats. J Neurosci 2005; 25 (18): 4550-4559.
18. Basso DM, Beattie MS, Bresnahan JC. A sensitive and reliable locomotor rating scale for open field testing in rats. J Neurotrauma 1995; 12: $1-21$.

19. Adas G, Arikan S, Karatepe $O$ et al. Mesenchymal stem cells improve the healing of ischemic colonic anastomoses (experimental study. Langenbecks Arch Surg 2011; 396: 115-126.

20. Yuksel S, Guleç MA, Gultekin MZ et al. Comparison of the early period effects of bone marrow-derived mesenchymal stem cells and plateletrich plasma on the Achilles tendon ruptures in rats. Connect Tissue Res 2016; 57 (5): 360-373.

21. Urdzíková LM, Růžička J, LaBagnara M et al. Human mesenchymal stem cells modulate inflammatory cytokines after spinal cord injury in rat. Int J Mol Sci 2014; 15 (7): 11275-11293.

22. Ruzicka J, Machova-Urdzikova L, Gillick $\mathbf{J}$ et al. A Comparative Study of Three Different Types of Stem Cells for Treatment of Rat Spinal Cord Injury. Cell Transplant 2017; 26 (4): 585-603.

23. Mukaino M, Nakamura M, Okada S, Toyama Y, Liu M, Okano H. Role of IL-6 in regulation of inflammation and stem cell differentiation in CNS trauma. Nihon Rinsho Meneki Gakkai Kaishi 2008; 31 (2): 93-98.

24. Nakamura M, Okada S, Toyama Y, Okano H. Role of IL-6 in spinal cord injury in a mouse model. Clin Rev Allergy Immunol 2005; 28 (3): 197-204.

25. Arima H, Hanada M, Hayasaka T et al. Blockade of IL-6 signaling by MR16-1 inhibits reduction of docosahexaenoic acidcontaining phosphatidylcholine levels in a mouse model of spinal cord injury. Neuroscience 2014; 269: 1-10.

26. Herrmann O, Tarabin V, Suzuki S et al. Regulation of body temperature and neuroprotection by endogenous interleukin- 6 in cerebral ischemia. J Cereb Blood Flow Metab 2003; 23 (4): 406-415.

27. Demircan PC, Sariboyaci AE, Unal ZS, Gacar G, Subasi C, Karaoz E. Immunoregulatory effects of human dental pulp-derived stem cells on T cells: comparison of transwell co-culture and mixed lymphocyte reaction systems. Cytotherapy 2011; 13 (10): 1205-1220.

28. Nosrat IV, Widenfalk J, Olson L, Nosrat CA. Dental pulp cells produce neurotrophic factors, interact with trigeminal neurons in vitro, and rescue motoneurons after spinal cord injury. Dev Biol 2001; 238: 120-132.

29. Yamamoto A, Sakai K, Matsubara K, Kano F, Ueda M. Review. Multifaceted neuro-regenerative activities of human dental pulp stem cells for functional recovery after spinal cord injury. Neurosci Res 2014; 78: $16-20$.

30. Sakai K, Yamamoto A, Matsubara $\mathrm{K}$ et al. Human dental pulp-derived stem cells promote locomotor recovery after complete transection of the rat spinal cord by multiple neuro-regenerative mechanisms. J Clin Invest. 2012; 122 (1): 80-90.

31. Leong WK, Henshall TL, Arthur A et al. Human adult dental pulp stem cells enhance poststroke functional recovery through non-neural replacement mechanisms. Stem Cells Transl Med 2012; 1: 177-187.

32. Crigler L, Robey RC, Asawachaicharn A, Gaupp D, Phinney DG. Human mesenchymal stem cell subpopulations express a variety of neuroregulatory molecules and promote neuronal cell survival and neuritogenesis. Exp Neurol 2006; 198: 54-64.

Received November 8, 2017. Accepted December 20, 2017. 